\title{
4 Behandlung von suizidalen und depressiven Symptomen und Störungen bei Patienten mit einer Borderline- Persönlichkeitsstörung im Jugendalter
}

Christian Fleischhaker und Eberhard Schulz

In Deutschland ist Tod durch Suizid die zweithäufigste Todesursache im Jugendalter. Als Risikofaktoren für vollendete Suizide werden impulsive Handlungsmuster, Selbstverletzungen, Depressionen, Sozialstörungen sowie frühkindlicher Missbrauch benannt. Die Haupthypothese der vorliegenden Untersuchung ist, dass sich Jugendliche mit suizidalen und depressiven Symptomen und einer sich entwickelnden Borderline-Persönlichkeitsstörung effektiv mit der verhaltenstherapeutisch orientierten Dialektisch-Behavioralen-Therapie (DBT) für Adoleszente behandeln lassen. Die DBT wurde von Linehan zur Behandlung von Frauen mit Borderline-Persönlichkeitsstörungen entwickelt, die entweder chronisch suizidal sind und/oder sich selber verletzen. Gegenüber der Standard-DBT wurde für die Arbeit mit Jugendlichen durch Miller \& Rathus eine Reihe von Modifikationen vorgenommen, um die Behandlung der jugendlichen Zielgruppe anzupassen. Durch unsere Arbeitsgruppe wurde die Dialektisch-Behaviorale Therapie für Adoleszente (DBT-A) für den deutschen Sprachraum überarbeitet und angepasst. Die Wirksamkeit der Therapie wird anhand eines Prä/Post-Vergleiches mit standardisierten Skalen zur Selbst- und Fremdeinschätzung evaluiert. Hierzu verwendeten wir unter anderem die SCL-9o-R, CBCL, YSR, ILK und CGI. In der vorliegenden 
4 Behandlung von suizidalen und depressiven Symptomen und Störungen

bei Patienten mit einer Borderline-Persönlichkeitsstörung im Jugendalter

Arbeit werden die Ergebnisse der DBT-A-Pilotstudie vorgestellt. Insbesondere depressive und suizidale Symptome bilden sich im Rahmen dieser Behandlung rasch zurück. Diese Therapieeffekte sind auch ein Jahr nach Therapieende stabil nachweisbar.

Aufgrund dieser viel versprechenden Ergebnisse befindet sich derzeit eine randomisierte multizentrische Studie in Planung.

\subsection{Schlüsselwörter}

Für die Problemgruppe suizidaler und sich selbstverletzender Jugendlicher mit zusätzlichen Symptomen einer instabilen Persönlichkeitsstörungen vom Borderline-Typ wurde seit Mitte der goer Jahre ein im Erwachsenenbereich gut etabliertes psychotherapeutisches Verfahren adaptiert und evaluiert (Miller 1999). Die dialektische behaviorale Therapie (DBT) (Linehan 1993a,b) wurde von Marsha Linehan für chronisch parasuizidale Frauen entwickelt, bei denen zusätzlich die Diagnose einer Borderlinestörung gestellt worden war. Parasuizidal ist hierbei definiert als jegliches akute, intendierte Verhalten, durch das eine physische Schädigung erfolgt, mit oder ohne der Intention zu sterben. DBT ist derzeit weiterhin das einzige empirisch begründete Therapieverfahren für suizidale Multiproblem-Patienten, dessen Wirksamkeit in 7 randomisierten Untersuchungen von vier verschiedenen Arbeitsgruppen nachgewiesen werden konnte (Lieb et al. 2004, Bohus et al. 2000a, Evans et al. 1999, Miller 1999, Linehan et al. 1991, 1994).

DBT für Adoleszente (DBT-A) wurde speziell für suizidale Jugendliche mit Persönlichkeitszügen einer instabilen Persönlichkeitsstörungen vom Borderline-Typ entwickelt, so dass DBT-A spezifisch sowohl an der Reduktion von suizidalen und parasuizidalen Verhaltensweisen arbeitet, als auch Strategien beinhaltet, wie die Patienten motiviert im Therapieprogramm gehalten und zur Mitarbeiten bewegt werden können.

DBT basiert auf der bio-psycho-sozialen Theorie von Linehan, die davon ausgeht, dass die Symptomatik durch eine pervasive emotionale Dysregulation verursacht wird, mit fehlender Passung zwischen einem Individuum, das aufgrund einer biologischen Verletzbarkeit Schwierigkeiten hat, seine Emotionen zu regulieren, und einer Umwelt, die diese Verletzlichkeit durch Unverständnis intensiviert. Vom theoretischen Konstrukt wird angenommen, dass diese Verhaltensweisen entstehen, wenn ein Kind mit Schwierigkeiten in der Emotionsregulation in einem invalidierenden Umfeld aufwächst. Invalidierend bedeutet in diesem Falle z.B., dass einem Kind chronisch und pervasiv mitgeteilt wird, dass die Verhaltensweisen des Kindes unsinnig, dumm und falsch sind. Die DBT betrachtet parasuizidale Verhaltensweisen daher als im unterschiedlichen Sinne funktional. Parasuizidales Verhalten ist hierbei häufig die einzige Möglichkeit der Patienten ihre Emotionen zu regulieren und Hilfe in einem ansonsten invalidierenden Umfeld zu erhalten. Aus Sicht der 
DBT sind parasuizidale Verhaltensweisen maladaptive Problemlösungen auf für die Patienten überwältigende, extrem intensive, schmerzhafte Emotionen. In diesem Kontext werden bei Jugendlichen häufig auch depressive Symptome beobachtet.

DBT-A für Jugendliche besteht aus einer Einzeltherapie, regelmäßigen Familiengesprächen, einem Fertigkeitentraining in der Gruppe unter Integration eines nahen Angehörigen, einer Telefonberatung durch den Einzeltherapeuten und einer Supervisionsgruppe. In der Abteilung für Psychiatrie und Psychotherapie im Kindes- und Jugendalter der Universität Freiburg wurde die amerikanische Version des DBT-A den deutschen Verhältnissen angepasst und übersetzt. Es wurde ein deutsches Therapiemanual erstellt (Böhme et al. 2002) und in der hier vorgestellten Pilotstudie evaluiert (Fleischhaker et al. 2005).

Die DBT für Jugendliche (DBT-A) besteht aus 16 wöchentlichen Einzel- und Gruppentherapiesitzungen (s. Abb. 1).

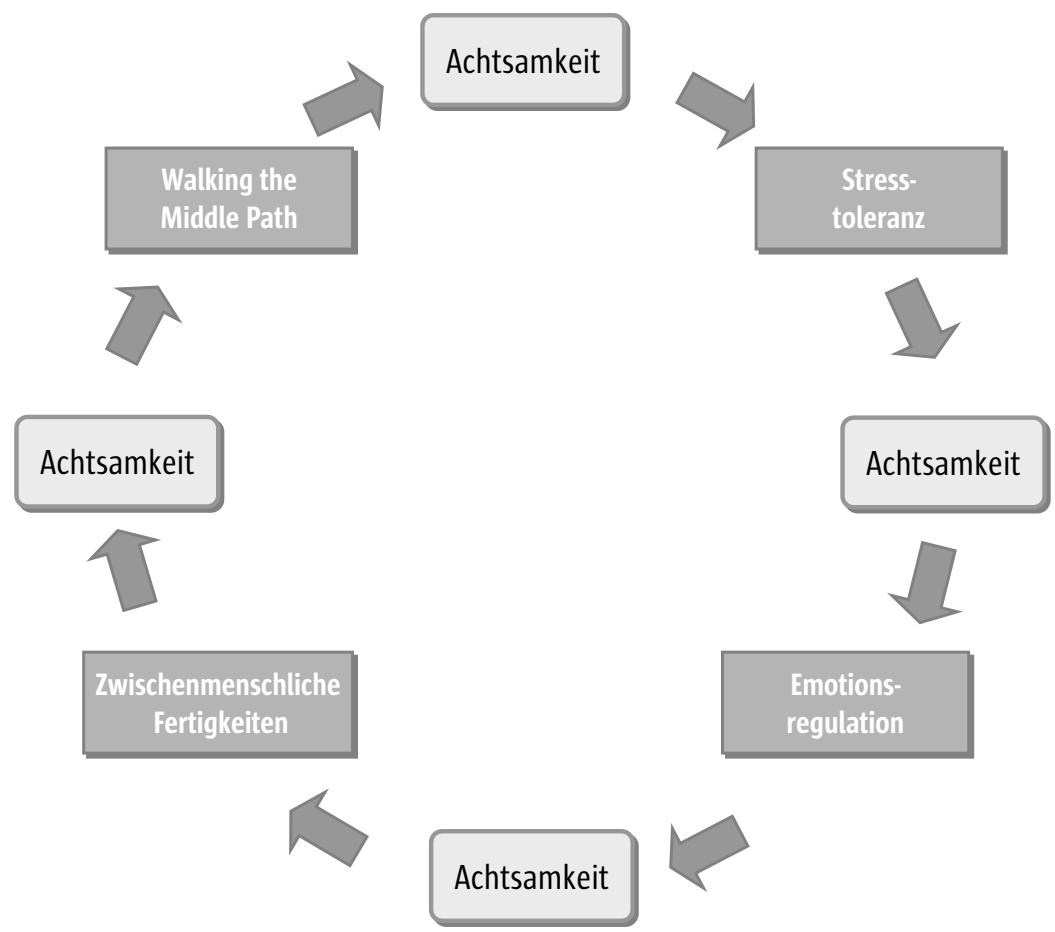

Abb. 1 Die halboffene Familien-Fertigkeiten-Trainingsgruppe gliedert sich in vier dreiwöchige spezifische Module (Stresstoleranz, Emotionsregulation, Zwischenmenschliche Fertigkeiten und Walking the Middle Path) und die einwöchigen Achtsamkeitsmodule, an denen neue Patienten einsteigen können. 
Der primäre Behandlungsfokus ist die Reduktion von suizidalen und selbstschädigenden Verhaltensweisen mit einem beständig wechselnden therapeutischen Fokus zwischen den Polen der Akzeptanz und der Veränderung. Der Therapeut oszilliert hierbei quasi zwischen problemorientierten Veränderungsstrategien (z.B. Verhaltensanalyse, Kontingenzmanagement zur Reaktionsverhinderung bzgl. heftiger Gefühle, kognitive Veränderungen von dysfunktionalen Gedanken und von dysfunktionalen Kommunikationsstategien) und akzeptierenden Strategien (z.B. Validierung des Patienten, direkter Intervention im Umfeld des Patienten).

Jugendliche mit suizidalen, parasuizidalen und depressiven Verhaltensweisen und vielen weiteren Schwierigkeiten benötigen eine effektive und störungsspezifische Behandlung, die dahingehend strukturiert ist, dass die Behandlung stets auf das Problem mit der höchsten Priorität systematisch fokussiert. Dies bedeutet in der Praxis, dass sich der Therapeut an einer dynamisch organisierten Hierarchie pathologischer Verhaltensmuster orientiert (Suizidversuche vor Gefährdung der Therapie vor Probleme der Lebensqualität). Zusammen mit dem Patienten erarbeitet der Therapeut zum jeweils hochrangigsten Problemverhalten Verhaltensanalysen und wählt diejenige Ebene aus (auslösende Faktoren, körperliche Reaktionen, emotionale Ebene, kognitive Ebene oder Ebene der Konsequenzen), die eine Wiederholung des Problemverhaltens am wahrscheinlichsten erscheinen lässt. Die gewählte Ebene zieht die entsprechende Behandlungsstrategie nach sich. Probleme in der Ebene der Anfälligkeitsfaktoren fordern in der Regel eine konkrete Problemlösung oder Verbesserung der zwischenmenschlichen Fertigkeiten. Körperliche Reaktionen lassen sich teilweise mit einer psychopharmakologischen Behandlung oder durch spezifische Fertigkeiten zur Affektmodulation und Stresstoleranz verändern. Die kognitive Ebene mit dysfunktionalen Gedankenschemata bedarf der Techniken der kognitiven Umstrukturierung und die Emotionsebene kann mit Expositionsübungen in Kombination mit einem geschickten Kontingenzmanagement bearbeitet werden. Die Schwierigkeit, die Compliance der Patienten zu erhalten und die Patienten zu Veränderungsprozessen zu motivieren, bedarf spezifischer Therapietechniken, die sowohl auf einer kontinuierlichen Validierung der Patientensichtweisen beruhen, als auch immer aktivierte konträre Schemata berücksichtigen sollte. Diese schwierige Balance von manifesten oder verborgenen Widersprüchen wird von Linehan als dialektische Strategie bezeichnet.

DBT für Jugendliche (DBT-A) bezieht in diese sensible Balance zwischen Akzeptanz bzw. Sinngebung von dysfunktionaler Verhaltensweisen einerseits und der Verdeutlichung der Notwendigkeit einer Veränderung andererseits eine Integration der Familie in das Behandlungskonzept mit ein. Dies geschieht sowohl im Fertigkeitentraining durch die Teilnahme eines Elternteiles, der die Fertigkeiten gleichberechtigt erlernt, als auch durch regelmäßige Integration der Eltern in die Einzeltherapie mit dem Ziel, hierbei jugendtümliche Dilemmata (z.B. Autonomieentwicklung versus Abhängigkeit) im jewei- 
ligen familiären Kontext zu fokussieren und dysfunktionale Familieninteraktionen zu bearbeiten. DBT-A kombiniert also Methoden wie Expositionsverfahren, kognitive Umstrukturierung, Problemlösetechniken, Vermittlung von Fertigkeiten und familientherapeutische Aspekte. Gerade das Vermitteln von neuen Fertigkeiten und Fertigkeitenketten beansprucht sehr viel Zeit auch in der Einzeltherapie und kollidiert damit häufig mit anderen therapeutischen Prozessen der Einzeltherapie, so dass eine gute Abstimmung und Prioritätensetzung in der Supervisionsgruppe unabdingbar ist. Aus diesem Grunde und aus zeitökonomischen und motivationalen Aspekten heraus erfolgt das Erlernen von spezifischen Fertigkeiten wie Stressregulation, Emotionsregulation, soziale Kompetenz, Achtsamkeit und „der mittlere Weg“ im Rahmen einer wöchentlich stattfindenden Gruppentherapie. Nach dem erfolgreichen Abschluss des 16-Wochenprogramms erhalten Patienten und Eltern ein Diplom für ihre erfolgreiche Teilnahme am Therapieprogramm. Auch die Einzeltherapie wird mit dem Ende des Gruppentherapieprogramms beendet, und der Jugendliche hat die Möglichkeit an einer Selbsthilfegruppe teilzunehmen, in der die Jugendlichen sich wöchentlich treffen, um darüber zu diskutieren, wie sie die neu erlernten Fertigkeiten effektiv einsetzen können, um ihre aktuellen Schwierigkeiten zu bewältigen. Diese Gruppe hilft den Jugendlichen die erlernten Fertigkeiten kontinuierlich einzusetzen und zu generalisieren und unterstützt die Jugendlichen gleichzeitig sich vom Therapeuten zu lösen. Die Arbeitsgruppe von Miller und Rathus konnte, sowohl in einem Prä/PostVergleich, als auch in einer Vergleichsstudie zu einer Standardbehandlung, die Effektivität des Behandlungsprogrammes für Jugendliche nachweisen (Rathus u. Miller 2002; Miller et al. 1997). Die evaluierten Veränderungen entsprechen weitgehend den therapeutischen Effekten der dialektischen behavioralen Therapie im Erwachsenenalter. DBT-A kann hierbei als Beispiel für eine sich entwickelnde, störungsspezifische, multimodale Behandlungsform gelten, die in manualisierter Form sowohl den Bedürfnissen dieser schwierigen Patientengruppe gerecht wird, als auch dem Therapeuten den notwendigen Rückhalt in einem Behandlungsteam liefert. Im Folgenden werden die Ergebnisse einer erst Pilotstudie zur deutschen Version der DBT-A vorgestellt.

\subsection{Erste Erfahrungen in einer Pilotstudie zur deutschen Version der DBT-A}

Die Pilotstudie zur deutschen Version der DBT-A an der Universität Freiburg zeigen ähnliche positive therapeutische Effekte (Fleischhaker et al. 2005), wie sie von Miller et al. berichtet werden (1997). In die vorliegende Therapiestudie konnten alle Patienten eingeschlossen werden, die die Ein- und Ausschlusskriterien erfüllten und die aufgrund der Entfernung zum Wohnort in der Lage waren, die ambulanten Therapietermine wahrzunehmen.

Die Pilotstudie wurde zunächst auf weibliche Patienten beschränkt, um eine größere Homogenität der Stichprobe zu gewährleisten. Aus pragmatischen 
4 Behandlung von suizidalen und depressiven Symptomen und Störungen

bei Patienten mit einer Borderline-Persönlichkeitsstörung im Jugendalter

und inhaltlichen Gründen wurden folgende Ein- und Ausschlusskriterien definiert:

\section{Einschlusskriterien}

- Alter zu Beginn der Therapie zwischen 13 und 19 Jahren

- Parasuizidale Verhaltensmuster in den letzten 16 Wochen und/oder aktuell bestehende Suizidgedanken

- Diagnose einer Borderline-Persönlichkeitsstörung oder Erfüllen von mindestens drei DSM-IV-Kriterien für eine Borderline-Persönlichkeitsstörung.

\section{Ausschlusskriterien}

- Kognitive Leistungsfähigkeit entsprechend einem Intelligenzquotienten (im CFT-20 oder HAWIK), der kleiner als 70 ist

- Aktuelle psychotische Erkrankung

- Aktuelle schwere depressive Episode oder Manie

- Suchterkrankung oder Essstörung als Erstdiagnose

- Deutliche Schwierigkeiten, zu lesen oder sich verbal auszudrücken

Im Rahmen der Gruppentherapie wurden 9 Patientinnen (75\%) von ihren Müttern begleitet. Bei den anderen drei Jugendlichen war es den Eltern aus unterschiedlichen Gründen nicht möglich an der Gruppentherapie teilzunehmen (z.B. Wohnort der Eltern mehr als $200 \mathrm{~km}$ entfernt, keine zeitlichen Ressourcen). Die Patientinnen waren bei Beginn der Behandlung im Mittel 16,25 Jahre alt (Standardabweichung: 1,75) mit einer Altersspanne zwischen 13 und 19 Jahren. Die kognitive Leistungsfähigkeit der Jugendlichen war mit einem Mittelwert des IQ von 112 (Standardabweichung: 14,6) und einer Spannweite zwischen 95 und 135 etwas in den oberen Bereich verschoben. Die durchgeführte standardisierte Diagnostik ergab bei jeder der behandelten Patientinnen mindestens eine Achse I Lebenszeit-Diagnose. Im Mittel wurden pro Patientin 2 Diagnosen vergeben (Spannweite: 1 bis 5). Am häufigsten fanden sich Neurotische und Belastungsstörungen (bei 66,7\% der Patienten), am zweithäufigsten affektive Störungen (50\%) gefolgt von Essstörungen (41,7\%) und Drogenabusus. Das Vorliegen von Persönlichkeitsstörungen wurde unter Verwendung des SKID-II evaluiert. Außer bei zwei Patientinnen ergab sich im SKID-II bei allen anderen Patientinnen mindestens eine Persönlichkeitsstörung. Bei 1o Patientinnen waren die Kriterien für das Vorliegen einer Borderlinestörung $(83,3 \%)$ erfüllt und bei 4 von diesen lag zusätzlich eine ängstlich (vermeidende) Persönlichkeitsstörung $(33,3 \%)$ vor.

Suizidversuche waren außer bei zwei Patientinnen bei allen in der Vorgeschichte zu eruieren. Bei allen Patientinnen lagen bei Beginn der Therapie Suizidgedanken vor. 
Selbstverletzendes Verhalten, zumeist im Sinne von sich Schneiden und Ritzen, war bei allen außer einer der behandelten Jugendlichen vorhanden.

Von den 12 Patientinnen, die das DBT-A Therapieprogramm begonnen haben, beendeten 9 Jugendliche $(75 \%)$ das Therapieprogramm vollständig.

Während und nach Abschluss des Therapieprogramms waren keine Suizidversuche zu verzeichnen. Selbstverletzende Verhaltensweisen reduzierten sich während der Behandlung signifikant. Das Globalniveau der psychosozialen Anpassung, erhoben anhand der GAF, verbesserte sich ebenfalls signifikant von vor Therapiebeginn (im Mittel 54) zu einem Monat nach Therapieende (im Mittel 76,9). Dieser positive Effekt bezogen auf die psychosoziale Anpassung bestätigt sich auch ein Jahr nach Therapieende (im Mittel 78,3).

Die Psychopathologie wurde unter anderem mittels der Symptom-Checkliste SCL-90-R gemessen. In der SCL-90-R gab es signifikante Verbesserungen unter der Therapie in den globalen Kennwerten Global Severity Index (Effektstärke $\mathrm{d}=1,38$; p-Wert des Wilcoxon-Tests = o,oo8), Positive Symptom Distress Index $(d=1,32 ; p=0,008)$ und Positve Symptom Total $(d=1,01 ; p=0,025)(s$. Abb. 2).

Die selbst berichtete Symptomatik der Jugendlichen reduzierte sich in den Skalen Zwanghaftigkeit $(d=1,08 ; p=0,012)$, Depressivität $(d=1,69 ; p=0,008)$, Ängstlichkeit $(\mathrm{d}=1,49 ; \mathrm{p}=0,012)$ und Aggressivität/Feindseligkeit $(\mathrm{d}=0,74$; $\mathrm{p}=\mathrm{o}, 035$ ). Bei den übrigen Skalen Somatisierung, Unsicherheit im Sozialkontakt, Phobische Angst, Paranoides Denken und Psychotizismus zeigten sich keine signifikanten Verbesserungen. Eine mögliche Erklärung dafür ist, dass insbesondere in den Skalen Somatisierung, Phobische Angst, Paranoides Den-

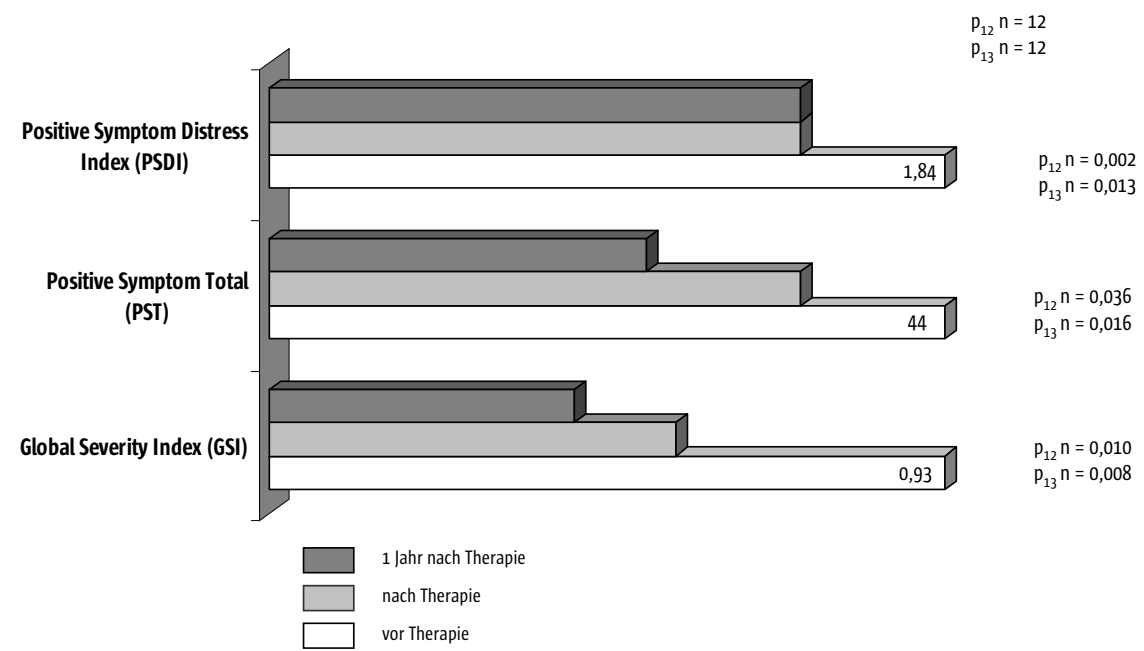

Abb. 2 Die Symptom-Checkliste SCL-90-R zeigt sowohl nach Therapie als auch bei der Katamnese ein Jahr nach Therapieende eine signifikante Verbesserung der Symptomatik. 
4 Behandlung von suizidalen und depressiven Symptomen und Störungen

bei Patienten mit einer Borderline-Persönlichkeitsstörung im Jugendalter

ken und Psychotizismus bei Therapiebeginn keine ausgeprägte Symptombelastung bestand.

In den genannten Subskalen bleibt die signifikante Verbesserung der Symptomatik auch bei der Nachuntersuchung ein Jahr nach Therapieende stabil bestehen. Insbesondere gilt dies auch für die depressive Symptomatik. Bei der Nachuntersuchung berichteten die Patientinnen, dass sie insbesondere von den Achtsamkeitsübungen und den Strategien zur Emotionsregulation profitiert hätten. Durch die konsequente Anwendung dieser Fertigkeiten sei es ihnen insbesondere gelungen depressive Symptome besser zu regulieren.

\subsection{Diskussion}

In der vorliegenden Untersuchung konnte gezeigt werden, dass die Dialektische Behaviorale Therapie für Adoleszente (DBT-A), auch in der durch die Arbeitsgruppe auf deutsche Verhältnisse angepassten Version, praktikabel durchgeführt werden kann. 75\% der Patienten schließen die Therapie ab und können somit während der gesamten Behandlungszeit in der Therapie gehalten werden. Dies entspricht den positiven Erfahrungen bezogen auf die Abbruchquoten aus der Behandlung von Erwachsenen mit DBT und zeigt die Überlegenheit der DBT diesbezüglich im Vergleich zur Standardbehandlung mit Abbruchquoten von über $50 \%$.

Die in dieser Arbeit gezeigte Reduktion der in der DBT-A definierten Zielsymptomatik (suizidale Verhaltensweisen, und selbstverletzendes Verhalten) und die gleichzeitige Verbesserung der Psychopathologie (einschließlich der depressiven Symptomatik) und des Globalniveaus der psychosozialen Anpassung sind sehr erfolgversprechend und bestätigen die vorliegenden Untersuchungen zur Wirksamkeit der DBT bei Erwachsenen und Jugendlichen (Linehan et al. 1991, 1994; Miller u. Glinski 200o).

Die DBT-A kann hierbei als Beispiel für eine sich entwickelnde, störungsspezifische, multimodale Behandlungsform gelten, die in manualisierter Form sowohl den Bedürfnissen dieser schwierigen Patientengruppe gerecht wird, als auch dem Therapeuten den notwendigen Rückhalt in einem Behandlungsteam liefert.

\section{Literatur}

Böhme R, Fleischhaker C, Mayer-Bruns F, Schulz E (2002) Dialektisch-Behaviorale Therapie für Jugendliche (DBT-A) - Therapiemanual. Abt. für Psychiatrie und Psychotherapie im Kindes- und Jugendalter, Universität Freiburg.

Bohus M, Haaf B, Stiglmayr C, Pohl U, Böhme R, Linehan M (2000a) Evaluation of inpatient dialectical-behavioral therapy for borderline personality disorder - a prospective study. Beh. Res. Ther. 38: 875-87.

Bohus M, Limberger M, Ebner U, Glocker FX, Schwarz B, Wernz M, Lieb K (2000b) Pain perception during self-reported distress and calmness in patients with borderline personality disorder and self-mutilating behavior. Psychiatry Res. 95: 251-60. 
Delmo C, Weiffenbach 0, Gabriel M, Stadler C, Pouska F (2000) Diagnostisches Interview. Kiddie-Sads-Present and Lifetime Version (5. Auflage der deutschen Forschungsversion). Frankfurt: Klinik für Psychiatrie und Psychotherapie des Kindes- und Jugendalters, Universität Frankfurt.

Döpfner M, Plück |, Bölte S, Lenz K, Melchers P, Heim K (1998a) Elternfragebogen über das Verhalten von Kindern und Jugendlichen. Deutsche Bearbeitung der Child Behavior Checklist ( $C B C L / 4-18)$, Einführung und Anleitung zur Handauswertung, 2. Auflage. Köln: Arbeitsgruppe Kinder, Jugend- und Familiendiagnostik (KJFD).

Döpfner M, Plück |, Bölte S, Lenz K, Melchers P, Heim K (1998b) Fragebogen für Jugendliche. Deutsche Bearbeitung des Youth Self-Report der Child Behavior Checklist (CBCL/4-18), Einführung und Anleitung zur Handauswertung, 2. Auflage. Köln: Arbeitsgruppe Kinder, Jugend- und Familiendiagnostik (KJFD).

Evans K, Tyrer P, Catalan I, Schmidt U, Davidson K, Dent I, Tata P, Thornton S, Barber I, Thompson S (1999) Manual-assisted cognitive-behaviour therapy (MACT): a randomized controlled trial of a brief intervention with bibliotherapy in the treatment of recurrent deliberate self-harm. Psychol. Med. 29: 19-25.

Fleischhaker C, Böhme R, Sixt B, Schulz E (2005) Suizidalität, Parasuizidalität und selbstverletzende Verhaltensweisen von Patientinnen mit Symptomen einer Borderlinestörung - Erste Daten einer Pilotstudie zur Dialektisch-Behavioralen Therapie für Adolescente (DBT-A). Kindheit \& Entwicklung 14: 112-127.

Franke GH (1995) Die Symptom-Checkliste von Derogatis - Deutsche Version - Manual. Göttingen: Beltz Test $\mathrm{GmbH}$.

Lieb K, Linehan M, Zanarini M, Bohus M (2004) Borderline Personality Disorder. Lancet (in press).

Linehan MM (1987) THI. Treatment History Interview. Seattle: University of Washington.

Linehan MM (1993a) Cognitive behavioral treatment of borderline personality disorder. Guilford Press, New York.

Linehan MM (1993b) Skills training manual for treating borderline personality disorder. Guilford Press, New York.

Linehan MM, Armstrong HE, Suarez A, Allmon D, Heard HL (1991) Cognitive-Behavioral Treatment of Chronically Parasuicidal Borderline Patients. Archive of General Psychiatry 48: 1060-1064.

Linehan MM, Comtois K (1994) LPC. Lifetime Parasuicide Count. Seattle: University of Washington.

Linehan MM, Tutek DA, Heard HL, Armstrong HE (1994) Interpersonal Outcome of Cognitive Behavioral Treatment for Chronically Suicidal Borderline Patients. American Journal of Psychiatry 151: 1771-1776.

Mattejat F, Jungmann J, Meusers M, Moik C, Schaff C, Schmidt M-H, Scholz M, Remschmidt H (1998) Das Inventar zur Erfassung der Lebensqualität bei Kindern und Jugendlichen (ILK), Manuskript. Marburg: Klinik für Kinder- und Jugendpsychiatrie und -psychotherapie, Phillips-Universität Marburg.

Miller AL (1999) Dialectical Behavior Therapy: A New Treatment Approach for Suicidal Adolescents, Am. J. of Psychotherapy 53: 413-417.

Miller AL, Glinski I (2000) Youth Suicidal Behavior: Assessment and Intervention. Journal of Clinical Psychology 56: 1131-1152.

Miller AL, Rathus JH, Linehan MM (1997) Dialectical behavior therapy adapted for suicidal adolescents, J. Pract. Psy. and Behav. Health 3: 78-86.

National Institute of Mental Health (1976) CGI. Clinical Global Impressions. In: Guy W, Bonato RR (Eds.) Manual for the EDCEU Assessement Battery, $2^{\text {nd }}$ Rev. Ed. Maryland: Chevy Chase.

Rathus JH, Miller AL (2002) Dialectical behavior therapy adapted for suicidal adolescents. Suicide and LifeThreatening Behavior 32: 146-157.

Saß H, Wittchen H-U, Zaudig M, Houben I (2003) Diagnostisches und Statistisches Manual Psychischer Störungen Textrevision DSM IV-TR. Göttingen, Bern, Toronto, Seattle: Hogrefe-Verlag. 